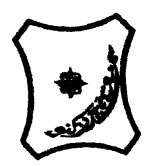

Bayero Journal of Pure and Applied Sciences, 12(2): 12 - 18

Received: July, 2019

Accepted: September, 2019

ISSN $2006-6996$

\title{
MULTIVARIATE GEOMETRIC AUTOREGRESSIVE AND AUTOREGRESSIVE MOVING AVERAGE MODELS
}

\author{
Umar, S. M.* and Bala, S. \\ Department of Mathematical Sciences, Bayero University, P.M.B. 3011, BUK, Kano- Nigeria. \\ * Corresponding author: surajoumar@yahoo.com
}

ABSTRACT

We present Autoregressive (AR) and autoregressive moving average (ARMA) processes with multivariate geometric (MG) distribution. The theory of positive dependence is used to show that in many cases, multivariate geometric autoregressive (MGAR) and multivariate autoregressive moving average (MGARMA) models consist of associated random variables. We also provide a special case of the multivariate geometric autoregressive model in which it is stationary and has multivariate geometric distribution.

Keywords: Multivariate geometric random vectors; Random diagonal matrix; Association; Joint stationarity; Characterization property; Marginal correlation.

\section{INTRODUCTION}

According to Block et al. (1988), a primary stationary model in time series analysis is the $P \times 1$ linear process given by

$$
X(n)=\sum_{j=-\infty}^{\infty} A(j) E(n-j), n=0, \pm 1, \pm 2,
$$

... (1.1)

where $A(j), j=0, \pm 1, \pm 2 \ldots$ is a sequence of $P \times P \quad$ parameter matrices such that $\sum_{j=-\infty}^{\infty}\|A(j)\|$ is finite and $E(n), n=0, \pm 1, \pm 2 \ldots$ is a sequence of independently and identically distributed $P \times 1$ random vectors with mean 0 and common covariance matrix. It is well known that equation (1.1) includes the stationary vector autoregressive (AR) process and the stationary and invertible vector autoregressive moving average (ARMA) process.

However, according to Lewis and Lawrence (1985), in some physical situations where the random vectors $X(n)$ are either positive or discrete, the preceding assumptions on the $E(n)$ sequence are inappropriate.

Langberg and Stoffer (1987) gives detail account of univariate geometric autoregressive (AR) and autoregressive moving average (ARMA) types of processes as well as the corresponding point processes in each case. Bivariate geometric autoregressive and autoregressive moving average models were designed by Block et al
(1988). In that paper, they present autoregressive and autoregressive moving average processes with bivariate geometric (BG) distribution. The theory of positive dependence is used by Block et al (1988) to show that in various cases, the bivariate geometric autoregressive (BGAR) and bivariate geometric autoregressive moving average (BGARMA) models consist of associated random variables. Special case of the bivariate geometric autoregressive process in which the bivariate process is stationary and has well known bivariate geometric distribution is also presented in Block et al (1988). Recently, Umar and Yusuf (2010), give autoregressive and autoregressive moving average models using multivariate exponential distribution. In that paper, they used theory of positive dependence to show that both autoregressive and autoregressive moving average of exponential distribution consist of associated random variables.

In this paper, we present rather classes of autoregressive and autoregressive moving average type sequences of multivariate random vectors. We use the theory of positive dependence to show that in many cases these classes of sequences are associated with each other. we also present the distribution under considerations, which is multivariate geometric distribution. Furthermore, we define the concept of association and present a variety of multivariate geometric distributions that are associated. Multivariate dependence mechanisms which are used in generating the various models are also discussed. 
BAJOPAS Volume 12 Number 2, December, 2019

We give the general multivariate geometric autoregressive $M G A R(m)$ model showing that the sequences have multivariate geometric distribution. Autocorrelation structure of the variety of sequences of the models is discussed. We consider special cases of sequences of the multivariate geometric autoregressive of order one, MGAR (1) where we show that if defined appropriately, the multivariate processes are stationeries and hence we obtain well-known multivariate geometric distribution. At the end,we provide multivariate geometric autoregressive moving average, $\operatorname{MGARMA}\left(m_{1}, m_{2}, \ldots, m_{k}\right)$ model.

\section{Some Definitions of Terms}

Definition 2.1: Let $M_{1}, M_{2}, \ldots, M_{k}$ berandom variables assuming values in the set $\{1,2,3, \ldots\}$. We say that $\left(M_{1}, M_{2}, \ldots, M_{k}\right)$ has a multivariate geometric distribution if each $M_{i}, \quad i=1,2, \ldots, k$, has geometric distribution.

Definition 2.2: Let $T=\left(T_{1}, T_{2}, \ldots, T_{n}\right), n=1,2, \ldots$, be a multivariate random vector. We say that random variables $T_{1}, T_{2}, \ldots, T_{n}$ are associated if $\operatorname{cov}(f(t), g(t)) \geq 0$ for all $f$ and $g$ monotonically non-decreasing in each argument, such that the expectations exist.

Remark 2.2: Note: From Barlow and Proschan (1981) and Block et al (1988), that independent random variables are associated.

Lemma 2.1: Let $\left(M_{1}, M_{2}, \ldots, M_{k}\right)$ and $\left(K_{1}, K_{2}, \ldots, K_{k}\right)$ be independent geometric random vectors such that for $k=1,2, \ldots, P\left(M_{i}=k\right)=\left(\frac{p_{i}}{\pi_{i}}\right)\left(1-\frac{p_{i}}{\pi_{i}}\right)^{k-1}$ and

$P\left(K_{i}=k\right)=p_{i}\left(1-p_{i}\right)^{k-1}, \quad i=1,2, \ldots, k$, with $0<p_{i}<1, \quad$ for all $i$. Let $\left(I_{1}, I_{2}, \ldots, I_{k}\right)$ be a multivariate Bernoulli random vector, which is independent of $\left(M_{1}, M_{2}, \ldots, M_{k}\right)$ and $\left(K_{1}, K_{2}, \ldots, K_{k}\right)$

. Then a random vector given by

$\left(G_{1}, G_{2}, \ldots, G_{k}\right) \underline{\underline{d}}\left(I_{1} K_{1}, I_{2} K_{2}, \ldots, I_{k} K_{k}\right)+\left(M_{1}, M_{2}, \ldots, M_{k}\right)$

has the same marginal distribution as $\left(K_{1}, K_{2}, \ldots, K_{k}\right)$.

The general multivariate geometric autoregressive model

In this section, we consider the construction of class of autoregressive (AR) sequence of multivariate random vector. This class of sequence is denotedby $\left\{G\left(m_{1}, m_{2}, \ldots, m_{k}\right)=G_{1}\left(m_{1}, m_{2}, \ldots, m_{k}\right), \ldots, G_{k}\left(m_{1}, m_{2}, \ldots, m_{k}\right)\right\} ; m_{i}=0,1,2, \ldots$ for all $i$. We

show that the random vector $\left(m_{1}, m_{2}, \ldots, m_{k}\right)$ has multivariate geometric distribution with mean vector that does not depend on $m_{1}, m_{2}, \ldots$ or $m_{k}$. Later, we discuss the association property for any finite number of random variables belonging to this autoregressive class. Finally, in this section we discussed the autocorrelation structure of this class of sequence.

For us to construct the class of multivariate geometric autoregressive sequence, some notations are needed.

Notations 3.1: Let $p_{1}, p_{2}, \ldots, p_{k}, \alpha_{1}, \ldots, \alpha_{k} \in(0,1)$ such that $p_{i} \leq \alpha_{i}(n)$, let $N^{\prime}(n)=\left(N_{1}(n), N_{2}(n), \ldots, N_{k}(n)\right)$ be a sequence of independent multivariate geometric random vectors with mean vectors $\left(\alpha_{1}(n) p_{1}^{-1}, \alpha_{2}(n) p_{2}^{-1}, \ldots, \alpha_{k}(n) p_{k}^{-1}\right)$ respectively, and let $M^{\prime}(n)=\left(M_{1}(n), M_{2}(n), \ldots, M_{k}(n)\right)$ be a sequence of independent multivariate geometric random vectors with common mean vector $\left(p_{1}^{-1}, p_{2}^{-1}, \ldots, p_{k}^{-1}\right)$ that are independent of $N^{\prime}(n)$. Also, let $J^{\prime}(n)=\left(J_{1}(n, 1), J_{1}(n, 2), \ldots, J_{1}(n, m), J_{2}(n, 1), \ldots, J_{k}(n, m)\right) \quad$ be $\quad$ a sequence of $k m-$ dimensional independent random vectors with components assuming the values 1 or 0 , independent of both $N^{\prime}(n)$ and $M^{\prime}(n)$. Let $C(n, q)$ be a $k \times k$ random diagonal matrix with 
BAJOPAS Volume 12 Number 2, December, 2019

$C(n, q)=\operatorname{diag}\left\{J_{1}(n, q), J_{2}(n, q), \ldots, J_{k}(n, q)\right\}, q=1,2, \ldots, m$. We also assume that for $l=1,2, \ldots, k$,

$\sum_{j=1}^{m} P\left\{\left(J_{1}(n, 1), J_{1}(n, 2), \ldots, J_{1}(n, m)\right)=e_{j}^{\prime}\right\}=1-\alpha_{1}(n)$

and that

$P\left\{\left(J_{l}(n, 1), J_{l}(n, 2), \ldots, J_{l}(n, m)\right)=e_{j}^{\prime}\right\}=\alpha_{l}(n)$.

We define the multivariate geometric autoregressive sequences as follows:

$G(n)= \begin{cases}M^{\prime}(n), & n=0,1,2, \ldots, m-1 \\ \sum_{q=1}^{m} C(n, q) G(n-q)+N^{\prime}(q), & n=m, m+1, \ldots .\end{cases}$

Next, we show that $G(n)$ has multivariate geometric distribution.

Lemma 3.1: Given that the variable $n$ takes on values $0,1,2, \ldots$, then $G(n)$ has a multivariate geometric distribution with mean vector $\left(p_{1}^{-1}, p_{2}^{-1}, \ldots, p_{k}^{-1}\right)$.

Proof: This lemma can be proved by an induction on $n$. For $n=0,1,2, \ldots, m-1$, the result of the lemma follow by the definition of $G(n)$. Let us assume that the result of the lemma holds for all non-negative integers that are less than or equal to $r, r \geq m-1$, and show that the result of the lemma holds for $r+1$.

Let $M^{\prime}=\left(M_{1}, M_{2}, \ldots, M_{k}\right)$ be a multivariate geometric random vector with mean vector $\left(p_{1}^{-1}, p_{2}^{-1}, \ldots, p_{k}^{-1}\right)$ independent of both $N^{\prime}(n)$ and $M^{\prime}(n)$. Then, by the induction assumption, we have for $l=1,2, \ldots, k$ that

$$
G_{l}(r+1) \underline{\underline{d}}\left\{\begin{array}{c}
M_{l}^{\prime}+N_{l}^{\prime}(r+1), \text { with probability } 1-\alpha_{l}(r+1) \\
N_{l}^{\prime}(r+1), \text { with probability } \alpha_{l}(r+1)
\end{array} .\right.
$$

It is easy to check that $G_{l}(r+1)$ has a geometric distribution with mean $p_{l}^{-1}$. Hence the result of the lemma follows. Therefore $G(n)$ has multivariate geometric distribution.

Next we consider the association of any finite collection of the $G_{l}(n)$ 's.

Lemma 3.2: Let us assume that for $j=0,1,2, \ldots, m-1$, the random variables $G_{1}(j), G_{2}(j), \ldots, G_{k}(j)$ in equation (3.3) are associated. Let $n_{1}<n_{2}<\ldots<n_{r}$ and $l_{1}, l_{2}, \ldots, l_{r}, \quad r=1,2, \ldots$, be positive integers. Then, the random variables $G_{l_{q}}\left(n_{q}\right), \quad q=1,2, \ldots, r$ are associated.

Proof: Let $T_{2 j}=M_{2}(j-1)$ and let $T_{2 j-1}=M_{1}(j-1), \quad j=1,2, \ldots$. To prove the result of lemma (3.2), it suffices, according to Barlow and Proschan (1981) and Jacob and Lewis (1983), to show that the random variables $T_{1}, T_{2}, \ldots, T_{r}$ are associated for all $r=1,2, \ldots \ldots$ (3.4)

We prove equation (3.4) by an induction argument on $r$. For $r \leq 2 m$, equation (3.4) follows by the lemma assumption and by Barlow and Proschan (1981). Let us assume that equation (3.4) holds for $r, r \geq 2 m$ and prove that equation (3.4) holds for $r+1$.

From equation (3.4), the conditional random variable $T_{r+1} / T_{1}, T_{2}, \ldots, T_{r}$, is stochastically nondecreasing in $T_{1}, T_{2}, \ldots, T_{r}$. Therefore, by Barlow and Proschan (1981), there is an $r+1$ argument function $h$, non-decreasing in each argument, and a random variable $U$ independent of $T_{1}, T_{2}, \ldots, T_{r}$, such that $\left(T_{1}, T_{2}, \ldots, T_{r+1}\right) \underline{\underline{d}}\left(T_{1}, T_{2}, \ldots, T_{r}, h\left(U, T_{1}, T_{2}, \ldots, T_{r}\right)\right)$. Hence $U$ is associated 
BAJOPAS Volume 12 Number 2, December, 2019

which implies the random variables $U, T_{1}, T_{2}, \ldots, T_{r}$ are also associated. Thus, $T_{1}, T_{2}, \ldots, T_{r+1}$ are associated.

Now, for this class of sequences, we proceed to compute the autocorrelation functions in the case when the marginal processes are stationary.

For the geometric models, substitute $\pi_{l}(n)=\pi_{l}$, for all $n ; l=1,2, \ldots, r$, and let $P\left\{I_{l}(n, q)=1\right\}=\Psi_{l}(q), \quad l=1,2, \ldots, k ; \quad q=1,2, \ldots, m \quad$ such $\quad$ that

(i) $\Psi_{l}(q) \geq 0$, and $($ ii $) \sum_{q=1}^{m} \Psi_{l}(q)=1-\pi_{l}, \quad l=1,2, \ldots, k$. Define

$\rho M_{l}(k)=\operatorname{Corr}\left\{M_{l}(n), M_{l}(n+k)\right\}, \quad l=1,2, \ldots, k ; \quad n=m, m+1, \ldots ; k=1,2, \ldots$.

Then

$\rho M_{l}(k)=\Psi_{l}(1) \rho M_{l}(k-1)+\Psi_{l}(2) \rho M_{l}(k-2)+\ldots+\Psi_{l}(m) \rho M_{l}(k-m)$

with Variance $\left\{G_{l}(n)\right\}=\left(1-p_{l}\right) p_{l}^{-2}, \quad l=1,2, \ldots, k$.

The marginal correlation structure of the multivariate geometric sequences, as given in (3.5) is similar to that of the Gaussian autoregressive process. We note that, in general, even when the marginal processes are stationary, the joint process is not stationary. This is easily seen, by letting $m=1$ in (3.3) with $\pi_{l}(n)=\pi_{l}$, for all $n ; l=1,2, \ldots, k$; choosing $G(n)$ to be an independently and identically distributed sequence of random vectors where $G_{1}(n), G_{2}(n), \ldots, G_{k}(n)$ are independently and identically distributed geometric random variables for all $n$, and letting $I(n)$ to be an independently and identically distributed sequence of random vectors for which $P\left\{I_{1}(n)=1, \ldots, I_{k}(n)=1\right\} \neq\left(1-\pi_{1}\right) \times\left(1-\pi_{2}\right) \ldots \times\left(1-\pi_{k}\right)$. A simple manipulation shows that $\operatorname{Cov}\left\{M_{1}(1), \ldots, M_{k}(1)\right\} \neq \operatorname{Cov}\left\{M_{1}(2), \ldots, M_{k}(2)\right\}$ in this example.

In the next section, we develop models in which joint processes are also stationary.

\section{Stationary multivariate geometric autoregressive model of order one [MGAR (1)]}

Here, we introduce special case of the multivariate geometric autoregressive of order $m$, in which the join processes are stationary. We consider the special case when $m=1$, assuming that $\alpha_{i}(n)$ does not vary with $n$ and put more structure on the $M(n)$ and $N(n)$ sequences. We show that for this model, the multivariate distribution of $G(n)$ has a form of the type studied by Arnold (1975), Block et al (1988) as well as Umar and Yusuf (2010). By selecting the $M(n)$ and $N(n)$ sequences as earlier defined, we can obtain well-known multivariate distributions. For a stationary MGAR(1) model, we obtain the: (i) Hawkes (1972) and (ii) Paulson-Uppuluri (1973) multivariate geometric distributions. We conclude this section by computing the auto covariance matrices for this model.

To develop stationary MGAR(1) model, we need to consider the following notations and assumptions. Let $m=1$ and let us assume that $\left(J_{1}(n, 1), \ldots, J_{k}(n, 1)\right)$, given in notation 3.1 , is an independently and identically distributed sequence of multivariate random vectors. Let us denote $\alpha_{l}(n)$ by $\alpha_{l}, l=1,2, \ldots, k$, and $P^{\prime}=P\left\{J_{1}(n, 1), \ldots, J_{k}(n, 1)\right\}$. Note that from equations (3.1) and (3.2), we have

$$
P_{10}+P_{11}=1-\alpha_{1}, \quad P_{01}+P_{11}=1-\alpha_{2} .
$$

Additionally, let $\left(Q_{1}, \ldots, Q_{k}\right)$ be a multivariate geometric random vector with parameters $P^{\prime}$ as given in equation (2.1). Let $p_{i} \in(0,1)$, for all $i$, such that $p_{l} \leq \alpha_{l}$, and let $N(r), r= \pm 1, \pm 2, \ldots$, be an independently and identically distributed sequence of multivariate geometric random vectors with mean vector $\left(\alpha_{1} p_{1}^{-1}, \ldots, \alpha_{k} p_{k}^{-1}\right)$ independent of $\left(Q_{1}, \ldots, Q_{k}\right)$ and all $\left\{J_{1}(n, 1), \ldots, J_{k}(n, 1)\right\}$. Note that 
BAJOPAS Volume 12 Number 2, December, 2019

by lemma (2.2), $\left(\sum_{j=1}^{Q_{1}} N_{1}(-j), \ldots, \sum_{j=1}^{Q_{k}} N_{k}(-j)\right)$ is a multivariate geometric random vector with mean vector $\left(p_{1}^{-1}, \ldots, p_{k}^{-1}\right)$. Now, let us assume

$M(0)=\left(\sum_{j=1}^{Q_{1}} N_{1}(-j), \ldots, \sum_{j=1}^{Q_{k}} N_{k}(-j)\right)$.

Define $C(n)$ to be the $k \times k$ diagonal random matrix $C(n)=\operatorname{diag}\left\{J_{1}(n, 1), \ldots, J_{k}(n, 1)\right\}$. The stationary $\operatorname{MGAR}(1)$ model is defined as follows:

$G(n)=\left\{\begin{array}{c}M(0), \quad n=0 \\ C(n) G(n-1)+N(n), \quad n=1,2, \ldots .\end{array}\right.$

Now, let us state and prove a characterization property of $G(n)$.

Lemma 4.1: Let $G(n)$ be defined as in equation (4.3) above. Then, for $n=0,1, \ldots, G(n) \underline{d} M(0)$, where $M(0)$ is as given in equation (4.2).

Proof: This lemma can be proved by an induction argument on $n$. By the definition $G(n)$, the result of this lemma holds for $n=0$. Let us assume that the result of this lemma holds for $n, n>0$ and show that it also holds for $n+1$. Note that

$$
\begin{aligned}
& M(0)=\left(\sum_{j=1}^{Q_{1}} N_{1}(-j), \ldots, \sum_{j=1}^{Q_{k}} N_{k}(-j)\right) \\
& \underline{\underline{d}}\left(\chi\left(N_{1}>1\right) \sum_{j=1}^{Q_{1}} M_{1}(-j), \ldots, \chi\left(N_{k}>1\right) \sum_{j=1}^{Q_{k}} M_{k}(-j)\right)+\left(N_{1}(-1), \ldots, N_{k}(-1)\right),
\end{aligned}
$$

where $\chi(\bullet)$ is the indicator function, and that $\mathrm{k}$ - summands are independent random vectors. By induction assumption,

$$
\begin{aligned}
& \left(\chi\left(N_{1}>1\right) \sum_{j=1}^{Q_{1}} M_{1}(-j), \ldots, \chi\left(N_{k}>1\right) \sum_{j=1}^{Q_{k}} M_{k}(-j)\right) \\
& \underline{\underline{d}}\left(J_{1}(n, 1) \sum_{j=1}^{Q_{1}} M_{1}(-j), \ldots, J_{k}(n, 1) \sum_{j=1}^{Q_{k}} M_{k}(-j)\right) \underline{\underline{d}}\left(J_{1}(n, 1) G_{1}(n), \ldots, J_{k}(n, 1) G_{k}(n)\right) .
\end{aligned}
$$

Furthermore, by the definition of $M(r),\left(M_{1}(-1), \ldots, M_{k}(-1)\right) \underline{\underline{d}}\left(M_{1}(n), \ldots, M_{k}(n)\right)$.

Sincethe random vectors $\left(M_{1}(n), \ldots, M_{k}(n)\right)$ and $\left(J_{1}(n, 1) G_{1}(n), \ldots, J_{k} G_{k}(n)\right)$ are independent, we have

$\left(\sum_{j=1}^{Q_{1}} M_{1}(-j), \ldots, \sum_{j=1}^{Q_{k}} M_{k}(-j)\right) \underline{\underline{d}}\left(M_{1}(n), \ldots, M_{k}(n)\right)+\left\{\left(J_{1}(n, 1) G_{1}(n), \ldots, J_{k}(n, 1) G_{k}(n)\right)=G^{\prime}(n+1)\right\}$.

Hence, the result of the lemma follows.

Finally in this section, we give the auto covariance matrix for the stationary MGAR(1) model. Let $\Sigma_{G}=\operatorname{Var}\{G(n)\}$ be the variance-covariance matrix of $G(n)$. Note that $\Sigma_{G}$ is independent of $n$ by lemma 4.1. Define $\Gamma_{G}(k)=\operatorname{Cov}\{G(n+k), G(n)\}, k=0,1,2, \ldots$, and note that $\Gamma_{G}(0)=\Sigma_{G}$. From equation (4.3), it is easy to see that $\Gamma_{G}(k)=C \Gamma_{G}(k-1), k=1,2, \ldots$, where $C$ is the $k \times k$ diagonal matrix defined by $C=\operatorname{diag}\left(1-\alpha_{1}, 1-\alpha_{2}, \ldots, 1-\alpha_{k}\right)$. Hence, for stationary MGAR(1) model, we have

$\Gamma_{G}(k)=C^{k} \Sigma_{G}, \Gamma_{G}(-k)=\Gamma_{G}^{\prime}(k), \quad k=0,1,2 \ldots(4.4)$ 
BAJOPAS Volume 12 Number 2, December, 2019

Multivariate geometric autoregressive moving average model

From the results obtained earlier and the one from Langberg and Stoffer (1987) for moving average sequences, we construct two classes of autoregressive moving average (ARMA) sequences of multivariate random vectors. In each class the sequences are labelled by the parameters $m_{1}, m_{2}, \ldots, m_{k}$. We denote these classes of sequences by $\left\{L^{\prime}\left(j, m_{1}, \ldots, m_{k}, n\right)=L_{1}\left(j, m_{1}, \ldots, m_{k}, n\right), \ldots, L_{k}\left(j, m_{1}, \ldots, m_{k}, n\right)\right\}, n=0,1, \ldots$, and $j=1,2, \ldots$ We show that the random vector $L^{\prime}\left(j, m_{1}, \ldots, m_{k}, n\right)$ has a multivariate geometric distribution with a mean vector that does not depend on $j, m_{1}, \ldots, m_{k}$ or $n$. Then, we discuss the association property of any finite number of random variables belonging to one of the two ARMA classes. Note that the parameters $m_{1}, m_{2}, \ldots$ and $m_{k}$ are fixed throughout this section.

Before the construction of the two classes of multivariate geometric autoregressive moving average (MGARMA) sequences, the following notations are needed.

Notation 5.1: Let $\delta_{1}, \ldots, \delta_{k}, \beta_{1}, \ldots, \beta_{k} \in(0,1)$ such that $\delta_{l} \leq \beta_{l}, l=1,2, \ldots, k$, and let $G(1, n), \ldots, G(k, n)$ be a MGAR sequence with mean vector $\left(\beta_{1} \delta_{1}^{-1}, \ldots, \beta_{k} \delta_{k}^{-1}\right)$. Also let $H(1, n), H(2, n), \ldots, H(k, n)$ be a dependent MGARMA sequence with mean vector $\left(\delta_{1}^{-1}, \ldots, \delta_{k}^{-1}\right)$ as given by Langberg and Stoffer (1987), be independent of all $G(1, n), \ldots, G(k, n)$. Finally, let $U^{\prime}(n)=\left(U_{1}(n), \ldots, U_{k}(n)\right)$ be an independently and identically distributed sequence of multivariate random vectors with components assuming the values 0or 1 independent of all the previous random vectors such that $P\left\{U_{l}(n)=1\right\}=1-\beta_{l}, \quad l=1,2, \ldots, k$. We define the two classes MGARMA sequences as follows:

$$
\begin{aligned}
& \left(L_{1}(1, n), \ldots, L_{k}(1, n)\right)=\left(G_{1}(1, n), \ldots, G_{k}(1, n)\right)+\left(U_{1}(n) H_{1}(1, n), \ldots, U_{k}(n) H_{k}(1, n)\right), \ldots(5.1) \\
& \left(L_{1}(2, n), \ldots, L_{k}(2, n)\right)=\left(H_{1}(2, n), \ldots, H_{k}(2, n)\right)+\left(U_{1}(n) G_{1}(2, n), \ldots, U_{k}(n) G_{k}(2, n)\right) .
\end{aligned}
$$

Next, we show that $L^{\prime}(j, n)$ has multivariate geometric distribution.

Lemma 5.1:For $j=1,2, \ldots$ and $n=0,1,2, \ldots, L^{\prime}(j, n)$ has a multivariate geometric distribution with mean vector $\left(\delta_{1}^{-1}, \delta_{2}^{-1}, \ldots, \delta_{k}^{-1}\right)$.

Proof: By lemma 3.1, $G(j, n)$ has a multivariate geometric distribution. Also by Langberg and Stoffer (1987), $H(j, n)$ has a multivariate geometric distribution. Therefore, the result of this lemma follows by the two above definitions as well as lemma 2.1 .

Now, we consider the association property of any finite number of random variables belonging to one of the two ARMA classes. We assume that the assumptions of Umar and Yusuf (2010) are true and lemma 3.2 is satisfied. We need to state and prove the following lemma.

Lemma 5.2: Let $S_{1}, \ldots, S_{r}, T_{1}, \ldots, T_{r}$ be non-negative random variables. Let us assume that $S_{1}, \ldots, S_{r}$ and $T_{1}, \ldots, T_{r}$ are associated, and that the random vectors $\left(S_{1}, \ldots, S_{r}\right)$ and $\left(T_{1}, \ldots, T_{r}\right)$ are independent. Then, the random variables $S_{1} T_{1}, \ldots, S_{r} T_{r}$ are associated.

Proof: Let $T^{\prime}=\left(T_{1}, \ldots, T_{r}\right)$, let $W=\left(S_{1} T_{1}, \ldots, S_{r} T_{r}\right)$ and let $f$ and $g$ be two non-negative functions each with $r$ arguments, non-decreasing in each argument.

The components of the conditional random vector $W / T^{\prime}$ are non-decreasing functions of the associated random variables $S_{1}, \ldots, S_{r}$. Therefore, by Barlow and Proschan (1981), the components of $W / T^{\prime}$ are associated. Hence,

$E\left[\operatorname{Cov}(f(W), g(W)) / T^{\prime}\right] \geq 0$. 
$E\left[f(W) / T^{\prime}\right]$ and $E\left[g(W) / T^{\prime}\right]$ are two non-decreasing functions of the associated random

variables $T_{1}, \ldots, T_{r}$. Thus, by Barlow and Proschan (1981), the two random variables $E\left[f(W) / T^{\prime}\right]$ and $E\left[g(W) / T^{\prime}\right]$ are associated. Thus, $\operatorname{Cov}\left[E\left\{f(W) / T^{\prime}\right\}, E\left\{g(W) / T^{\prime}\right\}\right] \geq 0$.

Note that

$$
\operatorname{Cov}[f(W), g(W)]=E\left[\operatorname{Cov}(f(W), g(W)) / T^{\prime}\right]+\operatorname{Cov}\left[E\left\{f(W) / T^{\prime}\right\}, E\left\{g(W) / T^{\prime}\right\}\right] \text {. }
$$

Therefore, this lemma follows. From this lemma, we can see the following result.

Lemma 5.3: Let us assume that $U_{1}(0), \ldots, U_{r}(0)$ are associated, let $n_{1}<n_{2}<\ldots<n_{r}$ and $l_{1}, \ldots, l_{r}, r=1,2, \ldots$, be as in lemma (3.2) above. Then, $L_{l_{q}}(1, n q), L_{l_{q}}(2, n q), \ldots, L_{l_{q}}(k, n q), q=1,2, \ldots, r$, are associated.

From the fore going, we can see that Langberg and Stoffer (1987) and section 4 of this work, give the inequalities and probability bounds for the multivariate point processes related to the multivariate geometric moving average sequences. We also note that all the results given by Block et al (1988) hold for the multivariate point processes related to the multivariate geometric autoregressive sequences, stated in sections 3 and 4, and to the autoregressive moving average sequences given in this section, provided that they are associated.

\section{CONCLUSION}

In this research work, We can conclude that Autoregressive (AR) and autoregressive moving

\section{REFERENCES}

Arnold, B.C (1975): A characterization of the exponential distribution by multivariate geometric compounding, Sankhya, A 37, 164173.

Barlow, R.E and Proschan, F (1981): Statistical Theory of reliability and life-testing: Probability Models. To begin with, Silver Spring, MD.

Block, H.W and Paulson, A.S. (1988): A note on finite divisibility of some bivariate exponential and geometric distributions arising from a compounding process. Sankhya 46, A, 102109.

Block, H.W, Langberg, N.A and Stoffer, D.S (1988): Bivariate exponential and geometric autoregressive and autoregressive moving average models. Advances in applied probability Trust, 20, No 4: 798-821.

Hawkes, A.G (1972): A bivariate exponential distribution with applications to reliability. J.R. Statist. Soc. B 34: 129-131. average (ARMA) processes with multivariate geometric (MG) distribution can be derived. The theory of positive dependence can be used to show that in many cases, multivariate geometric autoregressive (MGAR) and multivariate autoregressive moving average (MGARMA) models consist of associated random variables. In similar manner, a special case of the multivariate geometric autoregressive model in which it is stationary and has multivariate geometric distribution can be obtained.

\section{Acknowledgements}

The Authors are grateful to all those that contributed in whatever way towards the improved version of this paper.

Jacobs, P.A and Lewis, P.A.W (1983): Stationary discrete autoregressive moving average time series generated by mixtures. J. Times Series Anal. 4, 18-36.

Langberg, N.A. and Stoffer, D.S (1987): Moving average models with bivariate exponential and geometric distributions. J. Appl. Prob. 24, 4861.

Lawrence, A.J. and Lewis, P.A.W (1985): Modeling and residual analysis of nonlinear autoregressive time series in exponential variables J. R. Statist. Soc. B 47, 165-202.

Paulson, A.S. (1973): A characterization of the exponential distribution and a bivariate exponential distribution. Sankhya A 35, 69-78.

Umar, S.M and Yusuf, I (2010): Multivarite Exponential autoregressive and autoregressive moving average models. Journal of the Nig. Assoc. of Math. Physics. Vol.:16, 543-552. 